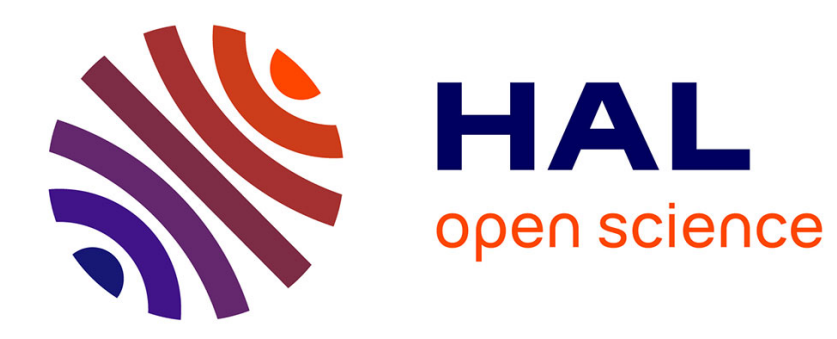

\title{
La sécheresse de 1949 en Haute-Bretagne
}

. de La Fouchardière

\section{- To cite this version:}

. de La Fouchardière. La sécheresse de 1949 en Haute-Bretagne. Revue forestière française, 1949, 6, pp.271-273. 10.4267/2042/27971 . hal-03536097

\section{HAL Id: hal-03536097 \\ https://hal.science/hal-03536097}

Submitted on 19 Jan 2022

HAL is a multi-disciplinary open access archive for the deposit and dissemination of scientific research documents, whether they are published or not. The documents may come from teaching and research institutions in France or abroad, or from public or private research centers.
L'archive ouverte pluridisciplinaire HAL, est destinée au dépôt et à la diffusion de documents scientifiques de niveau recherche, publiés ou non, émanant des établissements d'enseignement et de recherche français ou étrangers, des laboratoires publics ou privés. 


\title{
LA SÉCHERESSE DE 1949 EN HAUTE-BRETAGNE
}

\author{
Indice bibliographique : II.II.4: 42.22 (44.I)
}

L'année I949 comptera certainement dans les annales de la Météorologie comme une année exceptionnelle de sécheresse pour tout l'ensemble du territoire et particulièrement pour la région bretonne, bù nous avons pu réunir les observations suivantes:

La pluviosité annuelle (moyenne des 6o dernières années) est de $700 \mathrm{~m} / \mathrm{m}$ à Rennes. Or, depuis le mois d'août I948 jusqu'à fin juillet I949, cette pluviosité n'a été que de $4 \mathrm{II} \mathrm{m} / \mathrm{m} 5$; il saute donc aux yeux qu'il y a une réduction des $3 / 7$, réduction d'autant plus sensible que les années 1946, I947 et la première moitié de 1948 avaient été également des années sèches.

Ceci se manifeste aussi bien par le débit des rivières, tels que la Vilaine et le Couesnon qui ont pour habitude de déborder une ou deux fois par an, et qui depuis trois ans ne sont pas sortis de leur lit, que par l'abaissement de la nappe phréatique et un niveau anormalement bas des puits, des sources et des étangs.

Sur la végétation forestière, cette sécheresse a eu aussi des conséquences particulièrement remarquables, non seulement dans des jeunes peuplements ou dans des travaux de reboisement, mais également sur des arbres adultes vieux quelquefois d'un siècle et qui, en parfait état de végétation jusqu'à présent, semblent devoir périr complètement à la suite du manque d'eau.

La sécheresse actuelle est à la fois une sécheresse hivernale et une sécheresse estivale.

II est remarquable de constater la régularité avec laquelle sont. réparties les pluies dans la région bretonne. Dans le tableau suivant où nous faisons figurer en face de chaque mois d'abord le chiffre de la pluviosité moyenne (moyenne des 6o dernières années) et, en regard, le chiffre de la pluviosité pour le même mois au cours de 1948 et de 1949 .

$\begin{array}{cc}\text { Pluviosité en } \mathrm{m} / \mathrm{m} \\ \text { moyenne } & 48-49 \\ - & - \\ 53 & 57,5 \\ 56 & 44,8 \\ 75 & 48,8 \\ 88 & 38,2 \\ 58 & 72,4\end{array}$

$\begin{array}{llll}\text { Août } \ldots \ldots \ldots \ldots \ldots \ldots & 53 & 57,5 \\ \text { Septembre. } \ldots \ldots \ldots \ldots \ldots & 56 & 44,8 \\ \text { Octobre . . . } \ldots \ldots \ldots \ldots \ldots & 75 & 48,8 \\ \text { Novembre. } \ldots \ldots \ldots \ldots & 88 & 38,2 \\ \text { Décembre. } \ldots \ldots \ldots \ldots \ldots & 58 & 72,4\end{array}$




\begin{tabular}{|c|c|c|}
\hline Janvier & 66 & $2 \mathrm{I}, \mathrm{I}$ \\
\hline Février. . ............. & 62 & $\mathrm{I} 4, \mathrm{I}$ \\
\hline 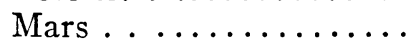 & 58 & 40,5 \\
\hline Avril . .............. & 52 & 20,5 \\
\hline Mai. ............. & 58 & 44,6 \\
\hline 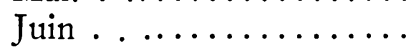 & 23 & 8 \\
\hline Juilleth $\ldots \ldots \ldots \ldots \ldots$ & :52 & $\mathbf{I}$ \\
\hline
\end{tabular}

Soit au total $70 I \mathrm{~m} / \mathrm{m}$ contre $4 \mathrm{II} \mathrm{m} / \mathrm{m} 5$.

Le mois d'août I948 a été légèrement plus pluvieux que la normale, avec $4 \mathrm{~m} / \mathrm{m} 5$ d'eau en supplément; de même le mois de décembre a présenté I $4 \mathrm{~m} / \mathrm{m}$ de plus que la pluviosité moyenne. Par contre, on voit tout de suite qu'à la fin de l'hiver, en janvier, février, mars et avril, on a eu une période exceptionnelle de sécheresse aggravée encore par l'action des vents qui ont été, à cette époque, assez violents et constamment des secteurs Nord et Est.

Néanmoins, au moment de la reprise de la végétation, rien n'a semblé anormal et ne pouvait laisser prévoir ce que l'on constate actuellement. Će n'est que depuis quelques semaines, à la suite de la très grande sécheresse du mois de juin et du mois de juillet (en ces deux mois il n'est tombé que $9 \mathrm{~m} / \mathrm{m}$ d'eau) que la végétation a commencé à souffrir, tout d'abord dans des plantations qui, bien souvent malgré toutes les précautions, ont été complètement anéanties.

De très nombreuses observations faites en ce sens, soit dans les forêts domaniales, soit au cours des contrôles de plantations subventionnées, ont permis de constater cet état de choses particulièrement lamentable.

Sur les grands arbres, à compter du $\mathrm{I}^{\text {er }}$ juillet, on a commencé à voir des signes de dépérissement, surtout hors forêts; là où les arbres sont en massifs ils n'ont pratiquement pas souffert, même dans des coupes secondaires de hêtres marquées assez claires, mais les plantations champêtres particulièrement abondantes en Bretagne, ont présenté cet aspect extraordinaire, à savoir qu'en de nombreux points des arbres adultes parfaitement vigoureux ont séché en quelques semaines, voire même en quelques jours et d'une façon qui ne laisse guère prévoir leur reprise.

Cette dessication a été très variable selon les essences et évidemment selon les terrains où elle se manifeste. L'arbre qui semble le plus atteint dans la plupart des cas est le Chataignier. Les taillis, surtout les jeunes taillis souffrent peu étant donné que pour une partie aérienne relativement réduite, ils ont des souches et des racines importantes, mais les arbres de haute tige ont séché, surtout dans les terrains filtrants, terrains sableux, terrains rocheux ou terrains possédant une déclivité notable. 
Cette dessication s'est faite, comme nous l'avons dit, d'une manière très rapide, très brutale: les feuilles ont blanchi sans avoir fané au préalable, et non seulement la feuille est actuellement morte, mais également des rameaux d'une grosseur allant jusqu'à celle du doigt; les bourgeons sont flétris, sont secs, et il est peu probable que des arbres aussi atteints puissent survivre.

Après le Châtaignier, c'est le Chêne et, chose paradoxale, le Bouleau pourtant très xérophile, qui ont souffert le plus, surtout sur les terrains sableux où évidemment ne pouvaient venir d'autres essences plus exigeantes en eau. L'aspect est sensiblement le même aue pour les Châtaigniers.

Le Hêtre qui demande pourtant beaucoup plus d'humidité atmosphérique a, en général, mieux résisté que les essences précitées. La dessication des hêtres ne s'est pas faite de la même façon; au lieu d'être brutale et caractérisée par le blanchissement des feuilles, cette dessication a été beaucoup plus lente et les feuilles ont roussi comme en automne. De plus, les bourgeons sont bien formés; le bois est resté vert, même sur les petits rameaux et l'arbre présente un aspect qui, au mois d'octobre, serait tout à fait anormal. Il y a de fortes chances pour qu'ils reprennent lorsque les conditions atmosphériques seront devenues plus favorables.

A part ces essences, les observations faites ne portent guère que sur des sujets isolés étant donnée la pauvreté générale de la flore forestière en Bretagne.

On peut cependant signaler qu'il n'y a pas encore été constaté un seul dessèchement sur les peupliers (à part dans les plantations de printemps), que de même des essences demandant en général beaucoup d'humidité, comme le Houx, n'ont pas souffert. Certains houx isolés pourtant sont restés parfaitement verts alors qu'à proximité immédiate des chênes sont en train de périr.

Sur les résineux, les effets de la sécheresse sont en général beaucoup moins graves. Dans les stations rocheuses cependant, que seuls pouvaient occuper les pins maritimes, ceux-ci ont souffert et seules les aiguilles des deux dernières années sont encore vertes.

Ces observations sont faites rapidement étant donné que l'été est tout juste commencé et que l'on ne peut préjuger de l'avenir. Il sera intéressant de les suivre au cours de l'été, de l'automne et du printemps prochain.

Il semble néanmoins bon de signaler dès maintenant les effets particuliers d'une sécheresse extraordinaire, car de mémoire d'homme, on n'avait vu ainsi des arbres isolés en pleine vigueur, des haies entières ou même de petits peuplements, sécher complètement en quelquues semaines.

DE LA Fouchardière. 